\title{
Immunomodulatory Effects of Mesenchymal Stem Cells and Mesenchymal Stem Cell-Derived Extracellular Vesicles in Rheumatoid Arthritis
}

\author{
Huan Liu ${ }^{1 \dagger}$, Ruicen Li $^{2+}$, Tao Liu ${ }^{1}$, Leiyi Yang ${ }^{1}$, Geng Yin ${ }^{1 *}$ and Qibing Xie ${ }^{1 *}$ \\ ${ }^{1}$ Department of Rheumatology and Immunology, West China Hospital, Sichuan University, Chengdu, China, ${ }^{2}$ Health \\ Management Center, West China Hospital, Sichuan University, Chengdu, China
}

OPEN ACCESS

Edited by:

Winston Patrick Kuo,

Harvard University, United States

Reviewed by:

Laura Mandik-Nayak,

Lankenau Institute for Medical

Research, United States

Kaoru Yamagata,

University of Occupational and Environmental Health Japan, Japan

${ }^{*}$ Correspondence:

Geng Yin

yingeng1975@163.com

Qibing Xie

xieqibing1971@163.com

${ }^{\dagger}$ These authors have contributed

equally to this work

Specialty section:

This article was submitted to Autoimmune and Autoinflammatory

Disorders,

a section of the journal

Frontiers in Immunology

Received: 27 March 2020

Accepted: 16 July 2020

Published: 20 August 2020

Citation:

Liu H, Li R, Liu T, Yang L, Yin G and Xie $Q$ (2020) Immunomodulatory Effects of Mesenchymal Stem Cells and Mesenchymal Stem Cell-Derived Extracellular Vesicles in Rheumatoid Arthritis. Front. Immunol. 11:1912.

doi: 10.3389/fimmu.2020.01912
Rheumatoid arthritis (RA) is a chronic autoimmune disease that affects the joints and other organs for which there is currently no effective treatment. Mesenchymal stem cells (MSCs) have therapeutic potential due to their immunomodulatory and differentiation effects. While extensive experimental studies and clinical trials have demonstrated the effects of MSCs in various diseases, MSCs have been found to cause abnormal differentiation and tumor formation. Therefore, extracellular vesicles derived from MSCs (MSC-EVs) are more effective, less toxic, and more stable than the parental cells. MSC-EVs transfer various nucleic acids, proteins, and lipids from parent cells to recipient cells, and thus participate in chronic inflammatory and immune processes. In this review, we summarize the properties and biological functions of MSCs and MSC-EVs in RA. Improvement in our understanding of the mechanisms underlying MSC and MSC-EVs in RA provides an insight into potential biomarkers and therapeutic strategies for RA.

Keywords: rheumatoid arthritis, mesenchymal stem cells, extracellular vesicles, exosomes, microRNAs

\section{INTRODUCTION}

Rheumatoid arthritis (RA) is a prevalent systemic autoimmune disease characterized by progressive joint destruction, and $50 \%$ of RA patients also have extra-articular involvement, including the heart, lungs, eyes, and blood $(1,2)$. Globally, the overall incidence of RA is 40/100,000 people per year, with a prevalence of $\sim 0.24 \%(3,4)$, which is significantly higher in women $(5)$. The etiologies and pathogenesis of RA have been extensively studied, wherein genetic susceptibility (i.e., HLA DR1, TRAF1, and STAT4), epigenetic modification (i.e., DNA methylation, miR146a, and miR155), and environmental factors (i.e., smoking, obesity, periodontitis, and vitamin $\mathrm{D}$ deficiency) have been found to promote the loss of immune tolerance, resulting in this disorder $(1,6-8)$. However, the precise mechanism underlying RA is complex and has not yet been elucidated. Currently, RA treatments, including glucocorticoid, immunosuppressants, and biological agents, are non-specific with an inadequate efficacy, severe adverse reactions, and even life-threatening toxic effects $(7,9)$.

Mesenchymal stem/stromal cells (MSCs) are a class of stem cells with self-renewal and multipotent properties that are widely available. As such, extensive clinical research has focused on the effects of MSCs in tissue regeneration and protection against injury via the replacement of damaged cells $(10,11)$. Subsequently, the evidence is increasingly indicating that MSCs play an 
immunomodulatory role primarily through the release of extracellular vesicles (EVs) and paracrine factors (e.g., growth factors, hormones, and cytokines) $(11,12)$. MSCs originate from many types of tissues, including bone marrow (BM), adipose tissue (AT), umbilical cord (UC), cord blood (CB), peripheral blood, dental pulp, liver, and the synovial membrane $(12,13)$. Generally, MSCs mostly express CD73, CD90, and CD105; however, these surface markers cannot be used to discriminate the source of MSCs. In contrast, MSCs negatively express CD14, CD34, CD45, and HLA-DR. MSCs can escape T cell recognition and exhibit low immunogenicity (14-17).

EVs are a group of lipid-bound vesicles that are released by various cells and play an essential role in the transfer of information between adjacent or distant cells. According to their origin, secretion mechanisms, and properties, EVs are divided into apoptotic bodies, microvesicles (MVs), and exosomes. Apoptotic bodies $(50-5,000 \mathrm{~nm})$ are released by dying cells into the extracellular space, and contain intact organelles, chromatin, and small amounts of glycosylated proteins. MVs (100$1,000 \mathrm{~nm}$ ) originate from plasma membranes. Exosomes (30$150 \mathrm{~nm}$ ) are formed by the intraluminal buds of multivesicular endosomes (MVEs) $(18,19)$. Due to the limitations of separation technologies, small EVs (sEVs) (50-200 nm) are commonly used in experimental studies (20). Among the different cells known to produce EVs, MSCs are one of the most prolific cells (21). Phenotypically, MSC-derived sEVs also express the MSC markers CD73, CD90, and CD105, but not CD14, CD34, or CD11b (17). The functions of MSC-EVs are similar to those of MSCs, although the latter are more stable, safe, less toxic, and are able to pass the blood-brain barrier, thus reducing their propensity to trigger immune responses (22-24). MSC-EVs transfer nucleic acids, including DNA, mRNA, and microRNA (miRNA); lipids; proteins; and surface receptors from donor cells to specific recipient cells, thereby protecting signaling molecules from enzymatic degradation during transport. MSC-EVs fuse with the recipient cell membrane either by directly fusing with the plasma membrane, fusing with the endosomal membrane after endocytosis, or by directly binding to the receptor of recipient cells, and then participate in physiological and pathological processes (25-27).

In recent years, studies have shown that MSCs and MSCEVs may be effective in RA, highlighting their potential immunomodulatory effects. In this review, we aim to discuss recent advances in the use of MSCs and MSC-EVs for the treatment of RA.

\section{IMMUNOMODULATORY EFFECT OF MSCS IN RA}

In the past decade, MSC transplantation (MSCT) has been found to be effective in the treatment of RA by reducing joint inflammation, bone erosion, and destruction and alleviating the formation of pannus via immune regulation, anti-inflammation, and differentiation $(28,29)$. MSCs mainly interact with both innate and adaptive immune cells to modulate immune responses in RA.
MSCs may regulate the proliferation, differentiation, and function of $\mathrm{T}$ cells and reduce the production of proinflammatory factors. In mouse models with collagen-induced arthritis (CIA), the administration of human AT-derived MSCs (AT-MSCs) inhibited the differentiation of activated CD4+ T cells into $\mathrm{T}$ helper (Th) 17 effector cells producing interleukin (IL)-17, but induced the generation of $\mathrm{T}$ regulatory cells (Tregs) that secrete IL-10 and negatively regulate the immune response (30). Similar beneficial effects have been reported in RA animal models using various MSC treatments $(28,31,32)$. The effects of MSCs on Th17/Treg cell balance have been attributed to various soluble molecules, including indoleamine 2,3-dioxygenase (IDO), IL-10, prostaglandin E2 (PGE2), and nitric oxide (NO), and to the transfer of organelles $(32,33)$. For example, after co-culturing healthy mice bone marrowderived MSCs (BM-MSCs) and Th17 from peripheral blood mononuclear cells (PBMCs) of RA patients, the proliferation of Th17 cells and production of IL-17 was inhibited by transferring mitochondria from BM-MSCs to Th17 cells. Simultaneously, mitochondrial transfer from the BM-MSCs of healthy donors was higher than that from the synovium-derived MSCs of RA patients (32). T follicular helper (Tfh) cells, a subset of CD4+ T cells, may help in immunoglobulin affinity maturation and generate live plasma cells and memory B cells $(34,35)$. Liu et al. found that the number of circulating Tfh cells increased, and was positively correlated with the disease and anti-cyclic citrullinated peptide antibody levels in RA patients (36). Subsequently, they further demonstrated that allogeneic UC-derived MSCs (UC-MSCs) suppressed the proliferation and function of Tfh cells via IDO production, which may be induced by interferon (IFN)- $\gamma$ in vivo and in vitro, thereby ameliorating the progression of CIA (37). Endoplasmic reticulum (ER)stressed MSCs could reduce the number of circulating Tfh cells via higher PGE2 binding with EP2/EP4 and increased IL-6 levels (38).

$\mathrm{B}$ cells mainly produce autoantibodies, including rheumatoid factor (RF) and anti-citrullinated protein antibodies (ACPAs), but also secret cytokines and act as antigen-presenting cells to promote T cell activation in RA (39). MSCs from healthy donors have been found to suppress B cell proliferation and anti-ACPA and RF production $(29,40)$. However, the mechanism underlying $\mathrm{B}$ cell regulation by MSCs in RA remains unclear. Currently, autologous MSCs injection has been considered to decrease B cell responses by reducing the levels of the B-cell activation factor (BAFF), a proliferation-inducing ligand (APRIL), and BAFF receptors (29). In comparison, in an in vitro experiment, BMMSCs from RA patients co-cultured with B cells from PBMC of healthy donors supported B cell survival, by a mechanism that may not be correlated with BAFF (41). This may be because of the conditional complexity of in vitro and in vivo experiments. In addition, the inhibition of MSCs on Tfh cells also indirectly affected the proliferation and differentiation of $\mathrm{B}$ cells (37).

Dendritic cells (DCs), macrophages, and natural killer (NK) cells are important members of the innate immune response and are regulated by MSCs in various diseases (42-44). However, their interaction with MSCs is scarcely studied in RA. Shin 


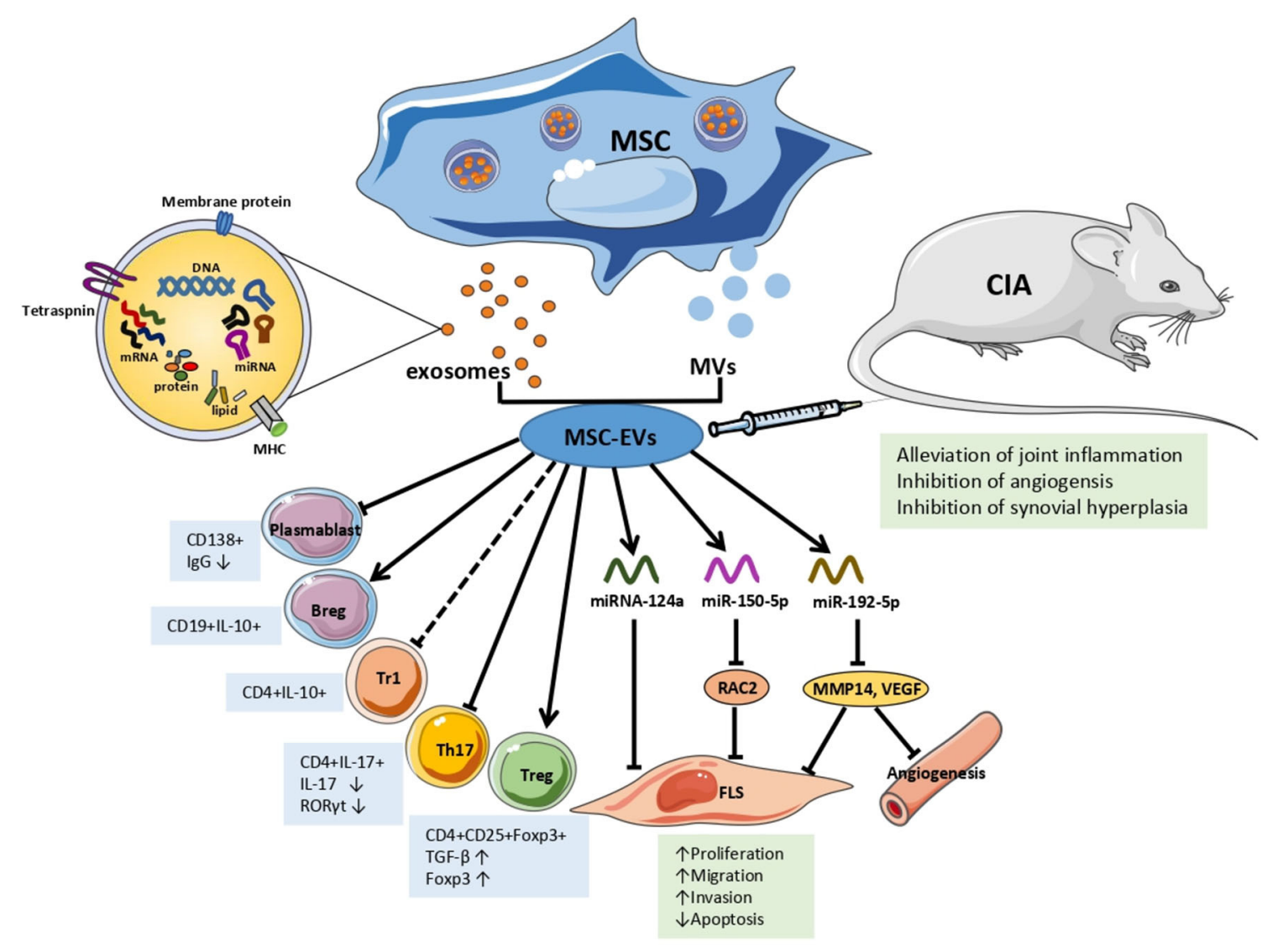

FIGURE 1 | The effects and mechanisms of MSC-EVs in RA. Arrows indicate activation or induction, T-bars indicate inhibition, dotted T-bars indicate inconsistent result, with Tr1 cells increasing in vitro but decreasing in vivo. MSC, mesenchymal Stem Cell; MVs, microvesicles; EVs, extracellular vesicles; Tr1, T regulatory type 1 cells; Th 17, T helper 17 effector cells; Treg, T regulatory cells; FLS, fibroblast-like synoviocytes; IgG, immunoglobulin G; TGF- $\beta$, transforming growth factor $\beta$; IL, interleukin; miRNA/miR, microRNA; RAC2, ras-related C3 botulinum toxin substrate 2; MMP14, matrix metalloproteinase 14; VEGF, vascular endothelial growth factor; CIA, collagen-induced arthritis mice model.

et al. demonstrated that MSCs inhibited the activation of M1type macrophages and induced the generation of M2-type macrophages via the tumor necrosis factor (TNF)- $\alpha$-mediated activation of cyclooxygenase-2 (COX-2) and TNF-stimulated gene-6. This was accompanied by the negative regulation of the nucleotide-binding domain, leucine-rich repeat pyrin 3 (NLRP3) inflammasome-mediated IL- $1 \beta$ secretion, and caspase1 production in macrophages through an IL-1 $\beta$ feedback loop (45). In addition, MSCs from systemic juvenile idiopathic arthritis patients were found to inhibit the differentiation of monocytes to DCs and suppress NK cell activation (46). Li et al. found that the combination of tolerogenic DCs and MSCs had a synergistic immunosuppressive effect on CIA mice by polarizing Th cells and inhibiting pro-inflammatory cytokines (47).

\section{CLINICAL MSC TRIALS IN RA}

In recent years, clinical research on the use of MSC therapy for the treatment of RA has increased. The first randomized clinical trial (RCT) using allogeneic expanded AT-MSCs (Cx611) for
RA treatment was conducted in 2011 as a multicenter, single blind, and placebo-controlled phase Ib/IIa clinical trial. A total of 53 refractory RA patients were enrolled and assigned to three cohorts with different doses $(1,2$, or 4 million cells/kg) and a placebo cohort, to evaluate the safety and tolerability of Cx611. The results indicated that the infusion of Cx611 was generally well-tolerated. One patient with dose-limiting toxicity (DLT) presented lacunar infarction. Most adverse events (AE) were mild or of moderate intensity. Although the most common symptoms were fever and infection, it was difficult to discern whether these were symptoms or simply side effects of Cx611 (48). In a phase Ia RCT investigating the efficacy and safety of the intravenous infusion of human CB-derived MSCs (hCBMSCs), 9 RA patients were divided equally among three groups, each receiving a single intravenous infusion of hCB-MSCs at different dosages. No short-term AE or DLT were reported 4 weeks after infusion. Moreover, the DAS 28 (28-joint disease activity score) was significantly decreased, pro-inflammatory cytokines were reduced, and IL-10 levels were increased $24 \mathrm{~h}$ after infusion (49). Similarly, a single-center RCT selected 30 RA 
patients with knee involvement to receive either intra-articular knee autologous BM-MSCT $(n=15)$ or normal saline $(n=$ 15). Following the transplantation of 40 million autologous BMMSCs, although no statistically significant results between the two groups were noted in the majority of the outcome measures, favorable effects on joint inflammation symptoms were observed, with an improved standing time in the MSCT group ( $p=$ $0.02)$. Moreover, MSCT treatment helped to reduce the dosage of MTX and prednisolone during the initial 6 months of followup, although not after 1 year. Importantly, no AEs were observed after MSC administration or during follow-up (50). Similarly, a clinical trial in Iran investigated whether injections of autologous BM-MSCs relieved the symptoms of refractory RA patients (51). These clinical trials found that MSC therapy for RA, especially refractory RA, is safe, well tolerable, and effective.

\section{IMMUNOMODULATORY EFFECTS OF MSC-EVS IN RA}

Recent studies have indicated that the mechanisms underlying the interaction between MSC-EVs and recipient cells are not unique in terms of their physiological or pathological processes in RA (Figure 1). Ma et al. found that both human UC-MSCs and the EVs secreted by them inhibited the proliferation of $\mathrm{T}$ cells, promoted $\mathrm{T}$ cell apoptosis, decreased $\mathrm{ROR} \gamma$ levels, increased Foxp3 levels, and regulated the balance of Treg/Th17 cells in in vitro and in vivo experiments, resulting in delayed radiological progression and synovial hyperplasia inhibition (52). Notably, the partial effect of MSC-EVs was different from that of parental MSCs in RA. MSC-exosomes increased the number of Treg, whereas MSCs did not. And MSCs were more capable of reducing the number of CD4+IFN- $\gamma+$ T lymphocytes. Compared with parental BM-MSCs and MVs, the exosomes increased the number of Treg cells. In addition, this study showed that MSC-exosomes inhibited plasmablasts but generated Breg cells (53).

Based on the fact that EVs are able to transfer information to recipient cells, subsequent studies characterized the mechanisms by which EVs, particularly through miRNAs, are involved in RA. Chen et al. were the first to report that BM-MSC-EVs transferred miR-150-5p to the joint cavity. Compared with osteoarthritis patients, the expression levels of miR-150-5p in the serum, synovial tissues, and fibroblast-like synoviocytes (FLS) of RA patients were significantly decreased, whereas the expression levels of matrix metalloproteinase (MMP) 14 and vascular endothelial growth factor (VEGF) were increased. MiR150-5p was effectively transfected into BM-MSCs in vitro and transferred by exosomes to RA-FLS. MSC-exosomal miR-150$5 \mathrm{p}$ suppressed the expression of the target genes MMP14 and VEGF by directly binding to their 3'-UTRs, thereby reversing the migration and invasion of RA-FLS and HUVEC tube formation induced by pro-inflammatory factors, including IL$1 \beta$, transforming growth factor $\beta$ (TGF- $\beta$ ), and TNF- $\alpha$. In vivo, the effect of MSC-exosome-miR-150-5p injection was consistent with those mentioned above, wherein MSC-exosome-miR-150$5 \mathrm{p}$ inhibited angiogenesis and alleviated joint inflammation (54).
Recently, miR-192-5p expression was found to be decreased in human RA-FLS, wherein a dual luciferase reporter gene assay showed that miR-192-5p directly targeted and negatively regulated ras-related $\mathrm{C} 3$ botulinum toxin substrate 2 (RAC2). In a CIA rat model, MSC-exosomal miR192-5p was transferred to the synovial tissue via the blood circulation after injection, and significantly reduced the levels of RAC2, decreased the clinical score, and suppressed synovial hyperplasia and joint destruction compared with rats injected with BM-MSCs-exosome-NC. Additionally, MSC-exosome-miR-192-5p inhibited the levels of pro-inflammatory cytokines, including PGE2, IL-1 $\beta$, and TNF$\alpha$, in synovial tissues and serum, and reduced the release of $\mathrm{NO}$ and inducible NO synthase (iNOS) in the sera of CIA rats (55). Another in vitro experiment found that the exosome number and miRNA-124a levels increased in MH7A cells (RAFLS cell lines) after co-culturing MH7A with human MSCEV. With miRNA-124a (hMSC-124a-EV) overexpression, the proliferation of MH7A was inhibited by hMSC-124a-EV and hMSC-EV compared with that in the control group. However, the cells were blocked in the G0/G1 and S phases, respectively. The invasion and migration of MH7A were also suppressed, while apoptosis was promoted. Moreover, the effect of hMSC-124a-EV treatment was more marked than that of hMSC-EV (56).

\section{PROSPECTS AND CHALLENGES IN THE CLINICAL APPLICATION OF MSCS AND MSC-EVS IN RA}

With an increasing number of studies, MSCs have been found to play an immunomodulatory role in numerous autoimmune diseases through the production of soluble factors, and the transfer of EVs containing messaging molecules (11, 57-59). In addition to immune regulation, MSCs can induce osteogenic and chondrogenic differentiation, and regulate inflammatory factors, highlighting it as a promising therapy for RA. Currently, most clinical trials of MSCT therapy for RA have focused on refractory RA patients who have not responded to traditional disease modifying antirheumatic drug (DMARDs) therapy, without any serious AEs associated with MSCT treatment. However, the use of MSCs in therapeutic treatments still faces many challenges. Several studies have found that MSCs are associated with carcinogenic risk when injected in animal models (60-63). Allogeneic MSCs have an immunosuppressive effect on tumor cells, allowing them to evade detection and destruction by the adaptive immune regulatory system via the action of CD8 $+\mathrm{T}$ cells, leading to the growth of allogeneic tumor cells (61). MSCs could also secrete VEGF to induce angiogenesis (62), contributing to tumor stroma formation, and favor tumor cell proliferation, invasion, and migration (60). The immunosuppressive effects of MSCs in CIA are also debatable. While MSCs can inhibit anti-CD3-induced T-cell proliferation in vitro, they do not affect $\mathrm{T}$ cell proliferation nor the development of CIA (64). Factors including the type of MSCs, culture conditions, treatment time, number of injected cells, injection route, and treatment regimen can lead to different results. A recent study compared the effects of three different types of 
MSCs infused into CIA mice and found that the most effective treatment was UC-MSCs, followed by BM-MSCs (65). The efficacy of allogeneic and autologous MSCs remains debatable, Rozier suggests that autologous MSCs may be involved in the physiopathology of systemic sclerosis (66). Therefore, RCT are necessary to compare the efficacy and safety of autologous and allogeneic MSC therapy in RA. BM-MSCs from RA patients were also found to promote Th17 cell activation and expansion via caspase 1 activation (67). In addition, different conditions also influence the effect of MSCs. For example, epigeneticallymodified MSCs (combination of hypomethylating agents and histone deacetylase inhibitors) have a high immunoregulatory effect in RA (68). Consequently, determining how long the immunomodulatory effects of MSCs last will need to be solved in clinical practice, the results of which could provide a theoretical basis and support for their use in the treatment of RA.

Compared with MSC treatment, which may cause abnormal differentiation and tumor formation, MSC-EVs are more effective, stable, and safer in alleviating inflammation of CIA, with broader prospects. EVs carry numerous DNAs, RNAs, proteins, and lipids from MSCs and transfer them to the recipient cells. Due to several advantages, including the ability to pass the blood-brain barrier and their low immunogenicity, EVs are natural carriers for drugs and exogenous nucleic acids, which can be loaded in donor cells before being released into the extracellular environment $(69,70)$. More importantly, using EVs to transfer miRNAs can prevent these from being degraded, allowing miRNAs to negatively regulate target protein expression at the post-transcriptional level. In addition to their treatment potential, several studies have reported that miRNAs secreted by MSC-EVs (MSC-EV-miRNAs) regulate diverse signaling pathways by targeting specific proteins, thereby influencing the development of RA. Therefore, MSC-EV-miRNAs are potential biomarkers for use in novel cell-free therapeutic strategies for RA.

Although MSC-EVs have been used in preclinical RA studies, several issues still remain unsolved. Firstly, when MSCs from different tissues are in distinct differentiation states, the content and types of molecules assembled by EVs may be different, thereby affecting their function at recipient cells and causing changes to physiological processes. MSC-EV miRNAs do not randomly enter EVs, however, the sorting mechanism by which

\section{REFERENCES}

1. Di D, Zhang L, Wu X, Leng R. Long-term exposure to outdoor air pollution and the risk of development of rheumatoid arthritis: a systematic review and meta-analysis. Semin Arthritis Rheum. (2020) 50:266-75. doi: 10.1016/j.semarthrit.2019.10.005

2. Wang D, Zhang J, Lau J, Wang S, Taneja V, Matteson EL, et al. Mechanisms of lung disease development in rheumatoid arthritis. Nat Rev Rheumatol. (2019) 15:581-96. doi: 10.1038/s41584-019-0275-x

3. Cross M, Smith E, Hoy D, Carmona L, Wolfe F, Vos T, et al. The global burden of rheumatoid arthritis: estimates from the global burden of disease 2010 study. Ann Rheum Dis. (2014) 73:1316-22. doi: 10.1136/annrheumdis-2013-204627

4. Ungprasert P, Srivali N, Cheungpasitporn W, Davis Iii JM. Risk of incident chronic obstructive pulmonary disease in patients with rheumatoid arthritis: cells are adjusted and selected from maternal cells is unknown. Generally, hundreds of differentially expressed miRNAs could be found in MSC-EVs by sequencing or microarray assays, however, no studies have performed miRNA expression profiling on MSC-EVs between RA and healthy individuals. Currently, dozens of miRNAs have been reported to affect the proliferation and function of FLS, previous studies also focused on the effect of MSC-EV-miRNAs on FLS, further studies on other cells are also needed. Additionally, whether the complex regulatory network of miRNAs and their target genes may trigger other diseases remains unclear and needs further study. Secondly, MSC-EVs secrete many other signaling molecules. Eirin et al. (71) integrated transcriptomic and proteomic analyses and found that the proteins, transcription factors, and translational regulators derived from MSC-EVs are involved in the mechanism of tissue repair in the recipient cell. Further studies on the interactions of the molecules that affect RA are necessary. Thirdly, EVs are separated in different ways, without standards, and in a time-consuming manner. Although commercial exosome extractants are currently being used, they contain non-exosome contaminants, such as lipoproteins, which need to be purified. Lastly, the findings presented here will need to be replicated on a large scale in clinical trials to assess the safety, effectiveness, and persistence of MSC-EVs in RA patients.

\section{AUTHOR CONTRIBUTIONS}

HL, RL, TL, and LY wrote sections of the manuscript. QX and GY critically revised the manuscript. QX reviewed and approved the version to be published. All authors contributed to manuscript revision, read, and approved the submitted version.

\section{FUNDING}

This research was supported by National Natural Science Foundation of China (Grant no. 30901339 and Grant no. 81172869), the Application Foundation Project in Sichuan Province (no. 2016JY0021 and no. 2017JY0025), and Clinical Research Incubation Project of West China Hospital, Sichuan University (no. 2019HXFH038). a systematic review and meta-analysis. Joint Bone Spine. (2016) 83:290-4. doi: 10.1016/j.jbspin.2015.05.016

5. Krasselt M, Baerwald C. Sex, symptom severity, and quality of life in rheumatology. Clin Rev Allergy Immunol. (2019) 56:346-61. doi: 10.1007/s12016-017-8631-6

6. Myasoedova E, Davis J, Matteson EL, Crowson CS. Is the epidemiology of rheumatoid arthritis changing? Results from a population-based incidence study, 1985-2014. Ann Rheum Dis. (2020) 79:440-4. doi: 10.1136/annrheumdis-2019-2 16694

7. Smolen JS, Aletaha D, McInnes IB. Rheumatoid arthritis. Lancet. (2016) 388:2023-38. doi: 10.1016/S0140-6736(16)30173-8

8. McInnes IB, Schett G. The pathogenesis of rheumatoid arthritis. $N$ Engl J Med. (2011) 365:2205-19. doi: 10.1056/NEJMra10 04965 
9. Moases Ghaffary E, Abtahi Froushani SM. Immunomodulatory benefits of mesenchymal stem cells treated with caffeine in adjuvant-induced arthritis. Life Sci. (2020) 246:117420. doi: 10.1016/j.lfs.2020.117420

10. Pittenger M, Vanguri P, Simonetti D, Young R. Adult mesenchymal stem cells: potential for muscle and tendon regeneration and use in gene therapy. $J$ Musculoskelet Neuronal Interact. (2002) 2:309-20.

11. Spees JL, Lee RH, Gregory CA. Mechanisms of mesenchymal stem/stromal cell function. Stem Cell Res Ther. (2016) 7:125. doi: 10.1186/s13287-016-0363-7

12. Li N, Hua J. Interactions between mesenchymal stem cells and the immune system. Cell Mol Life Sci. (2017) 74:2345-60. doi: 10.1007/s00018-017-2473-5

13. Tao SC, Yuan T, Zhang YL, Yin WJ, Guo SC, Zhang CQ. Exosomes derived from miR-140-5p-overexpressing human synovial mesenchymal stem cells enhance cartilage tissue regeneration and prevent osteoarthritis of the knee in a rat model. Theranostics. (2017) 7:180-95. doi: 10.7150/thno.17133

14. Li T, Xia M, Gao Y, Chen Y, Xu Y. Human umbilical cord mesenchymal stem cells: an overview of their potential in cell-based therapy. Expert Opin Biol Ther. (2015) 15:1293-306. doi: 10.1517/14712598.2015.1051528

15. Phinney DG, Galipeau J. Manufacturing mesenchymal stromal cells for clinical applications: a survey of good manufacturing practices at U.S. academic centers. Cytotherapy. (2019) 21:782-92. doi: $10.1016 /$ j.jcyt.2019.04.003

16. Haddouti EM, Randau TM, Hilgers C, Masson W, Walgenbach KJ, Pflugmacher R, et al. Characterization and comparison of human and ovine mesenchymal stromal cells from three corresponding sources. Int J Mol Sci. (2020) 21:2310. doi: 10.3390/ijms21072310

17. Witwer KW, Van Balkom BWM, Bruno S, Choo A, Dominici M, Gimona M, et al. Defining mesenchymal stromal cell (MSC)-derived small extracellular vesicles for therapeutic applications. J Extracell Vesicles. (2019) 8:1609206. doi: 10.1080/20013078.2019.1609206

18. Phelps J, Sanati-Nezhad A, Ungrin M, Duncan NA, Sen A. Bioprocessing of mesenchymal stem cells and their derivatives: toward cell-free therapeutics. Stem Cells Int. (2018) 2018:9415367. doi: 10.1155/2018/9415367

19. Doyle LM, Wang MZ. Overview of extracellular vesicles, their origin, composition, purpose, and methods for exosome isolation and analysis. Cells. (2019) 8:727. doi: 10.3390/cells8070727

20. Thery C, Witwer KW, Aikawa E, Alcaraz MJ, Anderson JD, Andriantsitohaina $\mathrm{R}$, et al. Minimal information for studies of extracellular vesicles 2018 (MISEV2018): a position statement of the International Society for Extracellular Vesicles and update of the MISEV2014 guidelines. J Extracell Vesicles. (2018) 7:1535750. doi: 10.1080/20013078.2018.1461450

21. Yeo RW, Lai RC, Zhang B, Tan SS, Yin Y, Teh BJ, et al. Mesenchymal stem cell: an efficient mass producer of exosomes for drug delivery. Adv Drug Deliv Rev. (2013) 65:336-41. doi: 10.1016/j.addr.2012.07.001

22. Lu Y, Zhou Y, Zhang R, Wen L, Wu K, Li Y, et al. Bone mesenchymal stem cell-derived extracellular vesicles promote recovery following spinal cord injury via improvement of the integrity of the blood-spinal cord barrier. Front Neurosci. (2019) 13:209. doi: 10.3389/fnins.2019.00209

23. Wu H, Fan $\mathrm{H}$, Shou $\mathrm{Z}$, Xu M, Chen Q, Ai C, et al. Extracellular vesicles containing miR-146a attenuate experimental colitis by targeting TRAF6 and IRAK1. Int Immunopharmacol. (2019) 68:204-12. doi: 10.1016/j.intimp.2018.12.043

24. Nojehdehi S, Soudi S, Hesampour A, Rasouli S, Soleimani M, Hashemi SM. Immunomodulatory effects of mesenchymal stem cell-derived exosomes on experimental type-1 autoimmune diabetes. J Cell Biochem. (2018) 119:943343. doi: $10.1002 /$ jcb. 27260

25. Mulcahy LA, Pink RC, Carter DR. Routes and mechanisms of extracellular vesicle uptake. J Extracell Vesicles. (2014) 3:24641. doi: 10.3402/jev.v3.24641

26. Raposo G, Stoorvogel W. Extracellular vesicles: exosomes, microvesicles, and friends. J Cell Biol. (2013) 200:373-83. doi: 10.1083/jcb.201211138

27. Mathivanan S, Ji H, Simpson RJ. Exosomes: extracellular organelles important in intercellular communication. J Proteomics. (2010) 73:1907-20. doi: 10.1016/j.jprot.2010.06.006

28. Yu Y, Yoon KA, Kang TW, Jeon HJ, Sim YB, Choe SH, et al. Therapeutic effect of long-interval repeated intravenous administration of human umbilical cord blood-derived mesenchymal stem cells in DBA/1 mice with collagen-induced arthritis. J Tissue Eng Regen Med. (2019) 13:1134-42. doi: 10.1002/term.2861
29. Gowhari Shabgah A, Shariati-Sarabi Z, Tavakkol-Afshari J, Ghasemi A, Ghoryani M, Mohammadi M. A significant decrease of BAFF, APRIL, and BAFF receptors following mesenchymal stem cell transplantation in patients with refractory rheumatoid arthritis. Gene. (2020) 732:144336. doi: 10.1016/j.gene.2020.144336

30. Zhou B, Yuan J, Zhou Y, Ghawji M Jr., Deng YP, et al. Administering human adipose-derived mesenchymal stem cells to prevent and treat experimental arthritis. Clin Immunol. (2011) 141:328-37. doi: 10.1016/j.clim.2011.08.014

31. Vasilev G, Ivanova M, Ivanova-Todorova E, Tumangelova-Yuzeir K, Krasimirova E, Stoilov R, et al. Secretory factors produced by adipose mesenchymal stem cells downregulate Th17 and increase Treg cells in peripheral blood mononuclear cells from rheumatoid arthritis patients. Rheumatol Int. (2019) 39:819-26. doi: 10.1007/s00296-019-04296-7

32. Luz-Crawford P, Hernandez J, Djouad F, Luque-Campos N, Caicedo A, Carrere-Kremer S, et al. Mesenchymal stem cell repression of Th17 cells is triggered by mitochondrial transfer. Stem Cell Res Ther. (2019) 10:232. doi: 10.1186/s13287-019-1307-9

33. Liu Y, Mu R, Wang S, Long L, Liu X, Li R, et al. Therapeutic potential of human umbilical cord mesenchymal stem cells in the treatment of rheumatoid arthritis. Arthritis Res Ther. (2010) 12:R210. doi: 10.1186/ar3187

34. Song W, Craft J. T follicular helper cell heterogeneity: time, space, and function. Immunol Rev. (2019) 288:85-96. doi: 10.1111/imr.12740

35. Wang J, Shan Y, Jiang Z, Feng J, Li C, Ma L, et al. High frequencies of activated $\mathrm{B}$ cells and $\mathrm{T}$ follicular helper cells are correlated with disease activity in patients with new-onset rheumatoid arthritis. Clin Exp Immunol. (2013) 174:212-20. doi: 10.1111/cei.12162

36. Liu R, Wu Q, Su D, Che N, Chen H, Geng L, et al. A regulatory effect of IL-21 on $\mathrm{T}$ follicular helper-like cell and B cell in rheumatoid arthritis. Arthritis Res Ther. (2012) 14:R255. doi: 10.1186/ar4100

37. Liu R, Li X, Zhang Z, Zhou M, Sun Y, Su D, et al. Allogeneic mesenchymal stem cells inhibited $\mathrm{T}$ follicular helper cell generation in rheumatoid arthritis. Sci Rep. (2015) 5:12777. doi: 10.1038/srep12777

38. Wei J, Ouyang X, Tang Y, Li H, Wang B, Ye Y, et al. ER-stressed MSC displayed more effective immunomodulation in RA CD4(+)CXCR5(+)ICOS $(+)$ follicular helper-like T cells through higher PGE2 binding with EP2/EP4. Mod Rheumatol. (2019) 30:509-16. doi: 10.1080/14397595.2019.1651446

39. Wang Q, Ma Y, Liu D, Zhang L, Wei W. The roles of B cells and their interactions with fibroblast-like synoviocytes in the pathogenesis of rheumatoid arthritis. Int Arch Allergy Immunol. (2011) 155:205-11. doi: $10.1159 / 000321185$

40. Usha Shalini P, Vidyasagar JV, Kona LK, Ponnana M, Chelluri LK. In vitro allogeneic immune cell response to mesenchymal stromal cells derived from human adipose in patients with rheumatoid arthritis. Cell Immunol. (2017) 314:18-25. doi: 10.1016/j.cellimm.2017.01.008

41. Dallos T, Krivosikova M, Chorazy-Massalska M, Warnawin E, Zanova E, Rudnicka W, et al. BAFF from bone marrow-derived mesenchymal stromal cells of rheumatoid arthritis patients improves their B-cell viabilitysupporting properties. Folia Biol. (2009) 55:166-76.

42. Shi B, Qi J, Yao G, Feng R, Zhang Z, Wang D, et al. Mesenchymal stem cell transplantation ameliorates Sjogren's syndrome via suppressing IL-12 production by dendritic cells. Stem Cell Res Ther. (2018) 9:308. doi: 10.1186/s13287-018-1023-x

43. Li JN, Li W, Cao LQ, Liu N, Zhang K. Efficacy of mesenchymal stem cells in the treatment of gastrointestinal malignancies. World J Gastrointest Oncol. (2020) 12:365-82. doi: 10.4251/wjgo.v12.i4.365

44. Zhou X, Jin N, Wang F, Chen B. Mesenchymal stem cells: a promising way in therapies of graft-versus-host disease. Cancer Cell Int. (2020) 20:114. doi: 10.1186/s12935-020-01193-Z

45. Shin TH, Kim HS, Kang TW, Lee BC, Lee HY, Kim YJ, et al. Human umbilical cord blood-stem cells direct macrophage polarization and block inflammasome activation to alleviate rheumatoid arthritis. Cell Death Dis. (2016) 7:e2524. doi: 10.1038/cddis.2016.442

46. Calkoen FG, Brinkman DM, Vervat C, van Ostaijen-Ten Dam MM, Ten Cate R, van Tol MJ, et al. Mesenchymal stromal cells isolated from children with systemic juvenile idiopathic arthritis suppress innate and adaptive immune responses. Cytotherapy. (2013) 15:280-91. doi: 10.1016/j.jcyt.2012. 10.017 
47. Li R, Zhang Y, Zheng X, Peng S, Yuan K, Zhang X, et al. Synergistic suppression of autoimmune arthritis through concurrent treatment with tolerogenic DC and MSC. Sci Rep. (2017) 7:43188. doi: 10.1038/srep43188

48. Alvaro-Gracia JM, Jover JA, Garcia-Vicuna R, Carreno L, Alonso A, Marsal S, et al. Intravenous administration of expanded allogeneic adiposederived mesenchymal stem cells in refractory rheumatoid arthritis (Cx611): results of a multicentre, dose escalation, randomised, single-blind, placebocontrolled phase Ib/IIa clinical trial. Ann Rheum Dis. (2017) 76:196-202. doi: 10.1136/annrheumdis-2015-208918

49. Park EH, Lim HS, Lee S, Roh K, Seo KW, Kang KS, et al. Intravenous infusion of umbilical cord blood-derived mesenchymal stem cells in rheumatoid arthritis: a phase Ia clinical trial. Stem Cells Transl Med. (2018) 7:636-42. doi: $10.1002 /$ sctm.18-0031

50. Shadmanfar S, Labibzadeh N, Emadedin M, Jaroughi N, Azimian V, Mardpour S, et al. Intra-articular knee implantation of autologous bone marrow-derived mesenchymal stromal cells in rheumatoid arthritis patients with knee involvement: results of a randomized, triple-blind, placebo-controlled phase 1/2 clinical trial. Cytotherapy. (2018) 20:499-506. doi: 10.1016/j.jcyt.2017.12.009

51. Ghoryani M, Shariati-Sarabi Z, Tavakkol-Afshari J, Ghasemi A, Poursamimi J, Mohammadi M. Amelioration of clinical symptoms of patients with refractory rheumatoid arthritis following treatment with autologous bone marrowderived mesenchymal stem cells: a successful clinical trial in Iran. Biomed Pharmacother. (2019) 109:1834-40. doi: 10.1016/j.biopha.2018.11.056

52. Ma D, Xu K, Zhang G, Liu Y, Gao J, Tian M, et al. Immunomodulatory effect of human umbilical cord mesenchymal stem cells on $\mathrm{T}$ lymphocytes in rheumatoid arthritis. Int Immunopharmacol. (2019) 74:105687. doi: 10.1016/j.intimp.2019.105687

53. Cosenza S, Toupet K, Maumus M, Luz-Crawford P, Blanc-Brude O, Jorgensen C, et al. Mesenchymal stem cells-derived exosomes are more immunosuppressive than microparticles in inflammatory arthritis. Theranostics. (2018) 8:1399-410. doi: 10.7150/thno.21072

54. Chen Z, Wang H, Xia Y, Yan F, Lu Y. Therapeutic potential of mesenchymal cell-derived miRNA-150-5p-expressing exosomes in rheumatoid arthritis mediated by the modulation of MMP14 and VEGF. J Immunol. (2018) 201:2472-82. doi: 10.4049/jimmunol.1800304

55. Zheng J, Zhu L, Iok In I, Chen Y, Jia N, Zhu W. Bone marrowderived mesenchymal stem cells-secreted exosomal microRNA-192-5p delays inflammatory response in rheumatoid arthritis. Int Immunopharmacol. (2020) 78:105985. doi: 10.1016/j.intimp.2019.105985

56. Meng HY, Chen LQ, Chen LH. The inhibition by human MSCsderived miRNA-124a overexpression exosomes in the proliferation and migration of rheumatoid arthritis-related fibroblast-like synoviocyte cell. BMC Musculoskelet Disord. (2020) 21:150. doi: 10.1186/s12891-020-3159-y

57. Kim HS, Choi DY, Yun SJ, Choi SM, Kang JW, Jung JW, et al. Proteomic analysis of microvesicles derived from human mesenchymal stem cells. $J$ Proteome Res. (2012) 11:839-49. doi: 10.1021/pr200682z

58. Qiu G, Zheng G, Ge M, Wang J, Huang R, Shu Q, et al. Mesenchymal stem cell-derived extracellular vesicles affect disease outcomes via transfer of microRNAs. Stem Cell Res Ther. (2018) 9:320. doi: 10.1186/s13287-018-1069-9

59. Mardpour S, Hamidieh AA, Taleahmad S, Sharifzad F, Taghikhani A, Baharvand $H$. Interaction between mesenchymal stromal cell-derived extracellular vesicles and immune cells by distinct protein content. J Cell Physiol. (2019) 234:8249-58. doi: 10.1002/jcp.27669

60. Djouad F, Bony C, Apparailly F, Louis-Plence P, Jorgensen C, Noel D. Earlier onset of syngeneic tumors in the presence of mesenchymal stem cells. Transplantation. (2006) 82:1060-6. doi: 10.1097/01.tp.0000236098.13804.0b
61. Djouad F, Plence P, Bony C, Tropel P, Apparailly F, Sany J, et al. Immunosuppressive effect of mesenchymal stem cells favors tumor growth in allogeneic animals. Blood. (2003) 102:3837-44. doi: 10.1182/blood-2003-04-1193

62. Xu S, Menu E, De Becker A, Van Camp B, Vanderkerken K, Van Riet I. Bone marrow-derived mesenchymal stromal cells are attracted by multiple myeloma cell-produced chemokine CCL25 and favor myeloma cell growth in vitro and in vivo. Stem Cells. (2012) 30:266-79. doi: 10.1002/stem.787

63. Zhu W, Xu W, Jiang R, Qian H, Chen $\mathrm{M}$, Hu J, et al. Mesenchymal stem cells derived from bone marrow favor tumor cell growth in vivo. Exp Mol Pathol. (2006) 80:267-74. doi: 10.1016/j.yexmp.2005. 07.004

64. Schurgers E, Kelchtermans H, Mitera T, Geboes L, Matthys P. Discrepancy between the in vitro and in vivo effects of murine mesenchymal stem cells on T-cell proliferation and collagen-induced arthritis. Arthritis Res Ther. (2010) 12:R31. doi: 10.1186/ar2939

65. Zhang Q, Li Q, Zhu J, Guo H, Zhai Q, Li B, et al. Comparison of therapeutic effects of different mesenchymal stem cells on rheumatoid arthritis in mice. PeerJ. (2019) 7:e7023. doi: 10.7717/peerj.7023

66. Rozier P, Maria A, Goulabchand R, Jorgensen C, Guilpain P, Noel D. Mesenchymal stem cells in systemic sclerosis: allogenic or autologous approaches for therapeutic use? Front Immunol. (2018) 9:2938. doi: 10.3389/fimmu.2018.02938

67. Eljaafari A, Tartelin ML, Aissaoui H, Chevrel G, Osta B, Lavocat F, et al. Bone marrow-derived and synovium-derived mesenchymal cells promote Th17 cell expansion and activation through caspase 1 activation: contribution to the chronicity of rheumatoid arthritis. Arthritis Rheum. (2012) 64:2147-57. doi: 10.1002/art.34391

68. Kim KW, Kim HJ, Kim BM, Kwon YR, Kim HR, Kim YJ. Epigenetic modification of mesenchymal stromal cells enhances their suppressive effects on the Th17 responses of cells from rheumatoid arthritis patients. Stem Cell Res Ther. (2018) 9:208. doi: 10.1186/s13287-018-0948-4

69. Zhang Y, Chopp M, Meng Y, Katakowski M, Xin H, Mahmood A, et al. Effect of exosomes derived from multipluripotent mesenchymal stromal cells on functional recovery and neurovascular plasticity in rats after traumatic brain injury. J Neurosurg. (2015) 122:856-67. doi: 10.3171/2014.11.JNS 14770

70. Vader P, Mol EA, Pasterkamp G, Schiffelers RM. Extracellular vesicles for drug delivery. Adv Drug Deliv Rev. (2016) 106:148-56. doi: 10.1016/j.addr.2016. 02.006

71. Eirin A, Zhu XY, Puranik AS, Woollard JR, Tang H, Dasari S, et al. Integrated transcriptomic and proteomic analysis of the molecular cargo of extracellular vesicles derived from porcine adipose tissue-derived mesenchymal stem cells. PloS ONE. (2017) 12:e0174303. doi: 10.1371/journal.pone.01 74303

Conflict of Interest: The authors declare that the research was conducted in the absence of any commercial or financial relationships that could be construed as a potential conflict of interest.

Copyright (C) $2020 \mathrm{Liu}, \mathrm{Li}$, Liu, Yang, Yin and Xie. This is an open-access article distributed under the terms of the Creative Commons Attribution License (CC BY). The use, distribution or reproduction in other forums is permitted, provided the original author(s) and the copyright owner(s) are credited and that the original publication in this journal is cited, in accordance with accepted academic practice. No use, distribution or reproduction is permitted which does not comply with these terms. 